\title{
Avaliação de Desempenho de Parques Tecnológicos: Proposta a Partir de um Estudo no TECNOPUC
}

\author{
Naiane de Lurdes Santana Bacharel em Administração de Empresas - Pontifícia Universidade Católica do Rio Grande do Sul (PUCRS) - Brasil \\ naiane-santana@hotmail.com (Brasil) \\ Peter Bent Hansen Doutor em Engenharia de Produção - Pontifícia Universidade Católica do Rio Grande do Sul (PUCRS) \\ peter.hansen@pucrs.br (Brasil)
}

\section{RESUMO}

Os parques tecnológicos vêm sendo considerados como valiosos instrumentos de promoção de diversos objetivos sociais e econômicos, cumprindo funções de suporte à integração entre agentes sociais. Considerando-se os ambientes dos parques, é relevante que se verifique a sua real efetividade como instrumentos de desenvolvimento tecnológico, competitivo e social. Assim, o objetivo deste trabalho é propor um sistema de indicadores que possibilite a avaliação de desempenho de parques tecnológicos, em especial os brasileiros. Esse sistema de indicadores foi desenvolvido com base em um estudo realizado no TECNOPUC (Parque Científico e Tecnológico da PUCRS) considerando a percepção dos seus principais stakeholders. Foram realizadas treze entrevistas com stakeholders (universidade, governo e empresas) atuantes e representantes no contexto dos parques no Rio Grande do Sul / Brasil, especificamente o TECNOPUC. Por meio das entrevistas foram identificados 10 objetivos estratégicos - associados a 4 dimensões (tecnológica, social, acadêmica e econômica) - e 29 indicadores considerados relevantes para avaliação de desempenho de parques científicos e tecnológicos. Esta pesquisa contribui para a identificação de dimensões que compilam os pontos essenciais de mensuração do desempenho de parques diante de uma estrutura de tríplice hélice que inclui universidade, governo e empresas.

Palavras-chave: avaliação de desempenho; parques científicos e tecnológicos; Stakeholders; Tecnopuc.

\section{Performance Evaluation of Technology Parks: Proposal Based on a Study at TECNOPUC}

\begin{abstract}
Technology parks are being considered as valuable tools to promote various social and economic objectives, fulfilling support functions for integration between social agents. Considering the environment of the parks, it is relevant to verify their actual effectiveness as instruments of technological, competitive and social development. The objective of this research is to propose a system of indicators that enables the performance evaluation of technological parks, especially the Brazilian parks. This system of indicators was developed based on a study conducted at TECNOPUC (Science and Technology Park of PUCRS) considering the perception of its main stakeholders. Thirteen interviews were made with stakeholders (university, government and companies) and active representatives in the context of the parks in Rio Grande do Sul / Brazil, specifically TECNOPUC. These interviews identified 10 strategic objectives - associated with 4 dimensions (technological, social, academic and economic) - and 29 indicators relevant to performance evaluation of science and technology parks. This research contributes to the identification of dimensions that compile the main points for performance measurement of technology parks based on a triple helix structure including university, government and business.
\end{abstract}

Keywords: performance evaluation; science and technology parks; stakeholders; Tecnopuc. 


\section{INTRODUÇÃO}

Nas últimas décadas, diversas transformações tecnológicas, sociais, políticas e econômicas vêm redesenhando o ambiente competitivo global e estabelecendo um cenário composto por novas exigências estratégicas em um meio organizacional mais incerto (Chikán, 2008). Neste contexto, os parques tecnológicos vêm sendo considerados como valiosos instrumentos de promoção de objetivos sociais, econômicos e políticos, cumprindo funções de suporte à integração entre vários agentes e gerando riquezas na Sociedade do Conhecimento (Spolidoro \& Audy, 2008; Jung Neto \& De Paula, 2009; Giugliani, Selig, \& dos Santos, 2012). Esses parques têm sido vistos como mecanismos de desenvolvimento local/regional, através do estímulo à inovação, competitividade e desempenho organizacional (Chan, Oerlemans, \& Pretorius, 2011; Campanella, Della Peruta, \& Del Giudice, 2014) e são reconhecidos como importantes componentes do Sistema Nacional de Ciência, Tecnologia e Inovação (Eler \& Andalecio, 2015; Guerra, Figueiredo, \& Ruscheinsky, 2015).

Considerando a complexidade do tema e partindo do pressuposto de que os parques tecnológicos no Brasil são relativamente recentes e que não existem muitos estudos voltados para a avaliação do desempenho de parques tecnológicos, coloca-se a seguinte questão norteadora desta pesquisa: Como avaliar o desempenho de um parque tecnológico considerando os diferentes enfoques dos stakeholders envolvidos no mesmo?

Assim, este trabalho apresenta o desenvolvimento de um sistema de indicadores que possibilite a avaliação de desempenho de parques tecnológicos, em especial, os brasileiros. Esse sistema de indicadores foi desenvolvido com base em um estudo realizado em um dos parques localizados no Rio Grande do Sul/Brasil - TECNOPUC (Parque Científico e Tecnológico da PUCRS) - considerando a percepção dos seus principais stakeholders.

Nas próximas seções deste artigo são apresentados os seguintes tópicos: (i) fundamentação teórica; (ii) método; (iii) resultados; (iii) conclusões; e (iv) referências.

\section{FUNDAMENTAÇÃO TEÓRICA}

Nesta seção será desenvolvida a fundamentação teórica do artigo, consistindo dos seguintes tópicos: (i) atores e objetivos vinculados aos parques tecnológicos; e (ii) medição de desempenho em parques tecnológicos.

\section{Atores e Objetivos Vinculados aos Parques Tecnológicos}

A conceituação e abordagem de parques tecnológicos desenvolvem-se em crescente proporção, de forma espontânea e estruturada, adaptando-se à realidade de cada país em que se localiza e possuindo diferentes objetivos. Parques tecnológicos são considerados e definidos como estruturas que propõem medidas e soluções significativas para as constantes mudanças frente à globalização e a nova sociedade do conhecimento (ABDI, 2008; Spolidoro \& Audy, 2008; Chiochetta, 2010; Giugliani, Selig, \& dos Santos, 2012).

Um parque tecnológico tem como propósito a relação entre a comunidade científica e a comunidade empresarial, possibilitando a união de conhecimentos e habilidades específicas de forma a proporcionar os seguintes resultados: (i) desenvolver a cultura da inovação e competitividade das empresas e instituições intensivas em conhecimento associadas ao parque; (ii) facilitar a transferência de tecnologia e habilidades empresariais entre academia e setor empresarial; (iii) estimular a criação e o desenvolvimento de empresas de base tecnológica por meio de incubadoras e spin-offs; (iv) promover o desenvolvimento de pesquisas científicas e tecnológicas; e (v) promover o desenvolvimento sustentável da comunidade e região em que está inserido (Spolidoro \& Audy, 2008).

Zouain e Plonski (2006) apontam que os conceitos e definições estão relacionados a diversos objetivos e fatores que, consequentemente, definem o tipo de parque. Assim, os tipos de parques tecnológicos podem ser categorizados em três formatos, sendo: (i) Parques tecnológicos - onde o ambiente é de união entre atividades de pesquisa e produção, sendo de vital importância o claro conhecimento dos gestores a respeito das necessidades da empresa em viabilizar atividades tanto de pesquisa quanto de produção integralmente; (ii) Parque científico e tecnológico - onde é forte o estímulo para haver sinergia entre universidades, empresas intensivas em conhecimento e centros de P\&D; e (iii) Parque tecnológico e empresarial - onde são oferecidos imóveis e serviços de suporte de alta qualidade aos atores e entidades residentes no parque (Spolidoro \& Audy, 2008). 
Existe uma diversidade de formatos de parques, que diferem em suas estruturas devido à expectativa e à orientação de cada ator engajado. Desta maneira, acredita-se que do mesmo modo que há uma diversidade de tipos de parques, há diversos atores/stakeholders que possuem objetivos diferentes e que impactam as estruturas dos parques tecnológicos influenciando no direcionamento estratégico dos mesmos e no seu modelo de atuação (Vedovello, 2000).

Considera-se no presente estudo a "tríplice hélice" de Etzkowitz e Leydesdorff (1995), que desenvolvem o modelo de interação entre Governo, Empresa e Universidade. Enfatiza-se a constante interação desses atores, em movimento de uma tríplice hélice, onde objetivos e características específicos dos mesmos são ferramentas para que se alcance o objetivo estabelecido pelo todo. $\mathrm{Na}$ tríplice hélice, a colaboração crescente entre os atores dos parques tecnológicos propicia o compartilhamento de informações e conhecimentos, viabilizando através disso o alcance de melhores resultados nos elos que conduzem o processo de pesquisa e inovação (Etzkowitz \& Leydesdorff, 1997).

Steiner, Cassim e Robazzi (2008) identificam para que uma política de inovação ocorra é necessário que se estabeleça uma forte parceria entre o setor governamental (financiamento público e impostos), o setor privado (onde se dá a transformação do conhecimento em riqueza) e a universidade (cuja missão é formar recursos humanos e produzir conhecimento). Deve ser ressaltado que cada pá da hélice (Universidade- Governo- Empresa) estabelece objetivos específicos por serem entidades de caráter distinto. Conforme Vedovello (2000) é através da interação de diversos propósitos que são estimulados e promovidos fortes laços.

Parques tecnológicos são definidos e valorizados como importantes propulsores do desenvolvimento tecnológico, econômico e social da região onde se inserem, sendo o objetivo fundamental dos parques o aumento da riqueza da comunidade envolvida e a promoção da cultura da inovação e da competitividade das empresas e instituições intensivas em conhecimento (Spolidoro \& Audy, 2008; Fernandes, 2014).

A Associação Internacional de Parques Tecnológicos (IASP, 2002) destaca que os principais meios de atingir os objetivos de um parque se verificam através de centros de incubação, prospecção de pesquisa científica e de desenvolvimento tecnológico e treinamento, os quais, com a interação de suas forças, fomentam uma economia que se desenvolve baseada em conhecimento.

No cenário mundial, parques tecnológicos têm sido instrumentos que inter-relacionam os vários agentes da economia, sendo direta ou indiretamente benéficos a todos os envolvidos (Atrasas, Souza Dias, \& Souza Leite, 2003). Segundo Chiochetta (2010), empreendimentos como parques tecnológicos têm por objetivo agregar ações que permitam acelerar o surgimento de produtos, processos e serviços nos quais a tecnologia assume o papel principal, além de gerar empregos e possibilitar a revitalização da região nos quais estão inseridos.

No cenário nacional, espera-se que os parques tecnológicos proponham soluções e melhorias inovadoras para problemas existentes na sociedade, enfocando no objetivo de potencializarem a vocação nacional e tornarem-se âncoras em projetos da sociedade (ABDI, 2008).

Para Etzkowitz (2009), existem nos parques tecnológicos três atores (stakeholders) que convergem para objetivos específicos, devido ao fato de cada ator envolvido manter uma identidade expectativa independente e distinta do outro. No Quadro 1, apresentam-se os objetivos específicos dos principais atores dos parques tecnológicos, em especial parques científicos e tecnológicos. Apesar de cada ator do parque possuir objetivos e expectativas diferentes, há uma interação e cooperação marcante na relação entre os atores (stakeholders), pois cada qual busca desenvolver melhorias no desempenho do outro (Etzkowitz, 2009). Diante disto, Etzkowitz e Leydesdorff (2000), abordam que por meio da interação dos objetivos de cada ator promove-se um objetivo em comum, o qual é estabelecer no parque um ambiente inovador composto por iniciativas trilaterais e alianças estratégicas entre os envolvidos. Assim, para Medori e Steeple (2000), as organizações e a estrutura do parque como um todo devem adotar e desenvolver medidas apropriadas, que analisem o seu desempenho quanto a cada objetivo estabelecido. Quando definidas as medidas de análise estas compõem um sistema de medição de desempenho, que poderá ser utilizado para visualizar os resultados com um nível maior de detalhes e especificidade (Esposto, Gerolamo, \& Rentes, 2002). 
Quadro 1. Objetivos dos Stakeholders de Parques Tecnológicos

\begin{tabular}{|c|c|}
\hline Stakeholders & Objetivos \\
\hline Governo & $\begin{array}{l}\text { - Beneficiar-se de alianças em nível regional, nacional e internacional } \\
\text { estimulando a formação de novas empresas de base tecnológica; } \\
\text { - Gerar novos postos de trabalho na região; } \\
\text { - Promover desenvolvimento econômico e social, melhorando a performance } \\
\text { da economia local; } \\
\text { - Reduzir os desequilíbrios regionais em termos de atividade de P\&D } \\
\text { (capacidade, investimento, inovação); } \\
\text { - Atrair investimento e atividade de P\&D; } \\
\text { - Melhorar a imagem das bases industriais locais, particularmente em regiões } \\
\text { economicamente deprimidas; e, } \\
\text { - Reproduzir o Vale do Silício e outras experiências bem-sucedidas. }\end{array}$ \\
\hline Empresa & $\begin{array}{l}\text { - Acessar os equipamentos e laboratórios universitários, tanto para produção } \\
\text { como para análise e testes; } \\
\text { - Recrutar estudantes, recém-graduados, bem como cientistas e engenheiros } \\
\text { mais experientes; } \\
\text { - Promover a atividade de consultoria por parte de acadêmicos; } \\
\text { - Estabelecer contratos de pesquisa e desenvolver, por meio da pesquisa, } \\
\text { produtos e serviços inovadores, promovendo também a interação entre os } \\
\text { Centros de Pesquisa; } \\
\text { - Encorajar o crescimento de novas empresas de base tecnológica que apenas } \\
\text { iniciaram suas atividades fora dos parques e incubadoras; } \\
\text { - Fomentar a sinergia entre as empresas para promover o benefício mútuo. }\end{array}$ \\
\hline Universidade & $\begin{array}{l}\text { - Encorajar e facilitar ligações entre a universidade e a indústria, envolvendo } \\
\text { prestação de serviços; } \\
\text { - Facilitar a transferência de tecnologia entre instituições acadêmicas e } \\
\text { empresas localizadas nesses empreendimentos; } \\
\text { - Comercializar pesquisa acadêmica; } \\
\text { - Aumentar a relevância, para a indústria, das pesquisas desenvolvidas por } \\
\text { universidades; } \\
\text { - Encorajar o surgimento de empresas spin-offs iniciadas por acadêmicos; } \\
\text { - Proporcionar às instituições acadêmicas acesso às atividades de P\&D de } \\
\text { ponta das empresas localizadas em parques e congêneres; } \\
\text { - Criar emprego e oportunidades de consultoria para pesquisadores e } \\
\text { estudantes; } \\
\text { - Gerar retorno financeiro para as instituições acadêmicas; } \\
\text { - Aumentar o conhecimento acadêmico a respeito das necessidades } \\
\text { industriais; e } \\
\text { - Melhorar a imagem das instituições acadêmicas junto ao governo. }\end{array}$ \\
\hline
\end{tabular}

Fonte: Elaboração própria, com base em Massey, Quintas e Wield (1992), Etzkowitz e Leydesdorff (2000) e Abdalla, Calvosa e Batista (2009)

Aborda-se na sequência uma breve discussão teórica sobre medição de desempenho em parques tecnológicos.

\section{Medição de Desempenho em Parques Tecnológicos}

A medição de desempenho pode ser definida como o ato de medir, congregando um conjunto de atividades, pressupostos e técnicas, que visam quantificar variáveis e atributos do objeto a ser analisado. Sua operacionalização ocorre através de indicadores (medidas) de desempenho, os quais em conjunto constituem um sistema de medição de desempenho (Kiyan, 2001).

Os indicadores de desempenho dependem essencialmente da orientação da organização, por isso as características organizacionais são fatores importantes para o desenvolvimento do modelo de medição a ser adotado (Spinola \& Pessôa, 1997). Desta forma, podem-se gerar indicadores teóricos, práticos, financeiros, estratégicos, táticos, operacionais, focado em processos, em pessoas e 
demais variações (Tezza, Bornia, \& Vey, 2010).

O sistema de medição de desempenho de parques tecnológicos surge preponderantemente da necessidade de avaliar os resultados obtidos no parque em si e, consequentemente, nas diversas empresas instaladas, tanto as recém-incubadas como as já desenvolvidas no mercado externo.

Diante da importância da aferição de resultados em parques tecnológicos e incubadoras (Chan, Oerlemans, \& Pretorius, 2011), indicadores de desempenho devem ser ajustados para que se adaptem aos vários enfoques, objetivos e atores existentes dentro dessa relação. A partir disso, os indicadores devem ser completos e expansivos para se avaliar essa estrutura, devido à complexidade de suas operações (Jung Neto \& De Paula, 2009).

Os indicadores que avaliam os parques tecnológicos são considerados de caráter diferenciado, pois cada tipo de organização e stakeholder envolvido têm missões e valores divergentes a serem aferidos. Para Galdámez, Carpinetti e Gerolamo (2009) os fatores que influenciam a medição de desempenho são as estruturas do próprio ambiente do parque, os quais são: (i) infraestrutura de gestão empresarial; (ii) nível de confiança; (iii) nível de cooperação; (iv) infraestrutura disponível para implantara prática; $\mathrm{e}$ (vi) diferentes interesses dos stakeholders. A partir disto, os autores desenvolveram um modelo de medição de desempenho no qual se destacam perspectivas de gerenciamento do processo de inovação, as quais são: (i) perspectiva econômica/ social, na qual se analisa o andamento da economia local, ocupação e capacitação dos trabalhadores; (ii) perspectiva ambiental, são apontados os desperdícios praticados pelas empresas e o impacto disso no meio-ambiente; (iii) perspectiva de desenvolvimento das empresas, que descreve o crescimento e a competitividade do ambiente em questão; (iv) perspectiva da eficiência coletiva, na qual são analisados os resultados obtidos por meio das ações coletivas planejadas e executadas pelas empresas inseridas na estrutura do parque; e (v) perspectiva de cooperação, é analisado o grau de confiança e cooperação praticado entre as empresas.

Numa direção semelhante, Ribeiro, Botelho e Duarte Filho (2012) utilizam a ferramenta Strategigram (estrategigrama) - implantada no Brasil pelos parques Porto Digital e Tecnopuc - que permite ter uma compreensão das ações estratégicas desenvolvidas em parques tecnológicos, fazendo comparações com outros parques e estruturas que utilizam esta ferramenta. Por meio dos resultados obtidos acredita-se que se pode reformular o planejamento fazendo ações corretivas (Mazzarolo, 2010). Na ferramenta "estrategigrama" são considerados sete eixos como vitais para a análise dos parques, onde se estabelecem posições para os eixos entre um índice de variância 10 e -10, os quais são: (i) localização do parque; (ii) uso da tecnologia, se criadora ou utilizadora de tecnologia; (iii) fomentação de empresas ou aperfeiçoamento das empresas existentes; (iv) foco em determinado ramo ou diversidade; (v) empresas locais ou de outras regiões e nacionalidades; (vi) avaliação do trabalho em redes e networking nos parques; (vii) escolha do modelo de gestão e governança dos centros tecnológicos.

Jung Neto e De Paula (2009) definem um conjunto de indicadores de desempenho para parques tecnológicos após um estudo de caso no Tecnopuc. Os autores estabeleceram medidas a partir da visão dos stakeholders do parque, e seguem a estrutura do modelo BSC (Balanced Scorecard). As dimensões elencadas são: (i) aspectos financeiros e sociais, que abordam benefícios de ordem social e econômica da localização que o parque está inserido; (ii) aspectos de gestão científica e tecnológica, os quais destacam a relevância de capacitar a gestão responsável pelo parque; e (iii) aspectos competitivos de infraestrutura e sustentabilidade, onde são medidos com um certo grau de dificuldade os fatores como produtividade da mão de obra e os acordos de cooperação de ordem internacional firmados por diferentes organizações no contexto do parque.

De acordo com Lima, Marinho e Carpinetti (2011), a medição de desempenho e seus respectivos indicadores devem refletir o desempenho da estrutura analisada como um todo, resultando em duas ações: (i) aumento da colaboração entre as empresas; e (ii) criação de ações conjuntas que venham a ter um impacto nas necessidades das concentrações. No contexto dos parques tecnológicos este é um desafio que merece atenção.

Na próxima seção deste artigo está detalhado o método de pesquisa utilizado.

\section{MÉTODO}

Este é um estudo do tipo exploratório e qualitativo de corte transversal (realizado em um determinado período do tempo). Trata-se de uma pesquisa exploratória porque, de fato, o tema medição de desempenho de parques tecnológicos é um assunto emergente e pouco verificado em estudos empíricos. Assim, um estudo exploratório é aconselhado quando os conhecimentos sobre 
o tema a ser abordado são poucos ou quando inexistem hipóteses desenvolvidas e testadas (Yin, 2015). A abordagem é qualitativa e livremente adaptada ao longo da sua execução, possibilitando constantemente um realinhamento da investigação e uma visão com detalhes minuciosos e bem particulares do pesquisador (Neves, 1996).

Para a coleta de dados, o instrumento elaborado e utilizado foi um roteiro de entrevista semiestruturado. Para aplicar o roteiro foram realizadas entrevistas presenciais com os stakeholders que fazem parte da estrutura da tríplice hélice, sendo atores do Governo, Universidade e Empresas. Tais atores foram escolhidos pelo conhecimento e contato direto com o ambiente de parques tecnológicos, mais especificamente o TECNOPUC.

O instrumento preliminar de pesquisa, o roteiro de entrevista, foi testado e ajustado a partir de uma Entrevista Piloto, realizada com um stakeholder da Universidade, que participa de uma unidade estratégica da gestão do parque. Este primeiro entrevistado é considerado o informante-chave na realização desta pesquisa, sendo este o primeiro contato prático desta pesquisa e também uma peça importante, uma vez que destacou e acompanhou todos os ajustes que se fizeram necessários no roteiro, antes da realização das entrevistas com os outros participantes.

As entrevistas, com a autorização dos entrevistados, foram gravadas para que posteriormente os seus conteúdos fossem transcritos e analisados. Foram realizadas 13 entrevistas, sendo (i) cinco empresas instaladas no parque Tecnopuc; (ii) quatro representantes da universidade que possuem cargos na Instituição e no ambiente do parque; e (iii) quatro representantes do governo que possuem cargos e atribuições de impacto direto no ambiente dos parques tecnológicos do Rio Grande do Sul - RS. Os entrevistados foram selecionados segundo a relação e expertise que possuem a respeito do tema estudado.

Para a análise dos dados utilizou-se algumas técnicas da análise de conteúdo, tais como a codificação, a classificação e a categorização de dados (Bardin, 2006). Os dados e as respectivas transcrições foram organizados e categorizados por meio do software NVivo de análise de dados qualitativos, o que viabilizou uma análise comparativa dos resultados de forma clara e objetiva. Os resultados dessas análises estão apresentados e discutidos a seguir.

\section{RESULTADOS}

Nesta seção são expostos os resultados obtidos através da realização desta pesquisa, consistindo principalmente em: (i) breve descrição do TECNOPUC e dos seus principais stakeholders; e (ii) proposta de um sistema de avaliação de desempenho para parques tecnológicos.

\section{Breve Descrição do TECNOPUC e dos seus Principais Stakeholders}

O TECNOPUC é o parque científico e tecnológico da PUCRS (Pontifícia Universidade Católica do Rio Grande do Sul), localizado em Porto Alegre/Brasil, cidade com uma população de 1,4 milhão de habitantes, possuindo uma região metropolitana com cerca de 3,6 milhões de habitantes.

Neste contexto situa-se o Parque Científico e Tecnológico da PUCRS - TECNOPUC, como resposta a uma demanda crescente do setor empresarial, que buscou na PUCRS a possibilidade de estabelecer parcerias que permitissem um crescimento mútuo.

A integração proposta no modelo de gestão do TECNOPUC começa com o envolvimento de empresas, avançando na busca do envolvimento de outros atores, como o governo e a universidade para a consolidação de uma rede de cooperação que contribua na promoção do desenvolvimento científico e tecnológico da cidade de Porto Alegre, da região e do país (Jung Neto \& De Paula, 2009). As principais características dos stakeholders do TECNOPUC que participaram desta pesquisa estão apresentadas no Quadro 2. 
Quadro 2. Principais características dos entrevistados: principais stakeholders do Tecnopuc

\begin{tabular}{|c|c|}
\hline Stakeholder & Características \\
\hline Universidade & $\begin{array}{l}\text { O stakeholder Universidade tem um papel estratégico e totalmente vinculado } \\
\text { à visão do Parque Tecnológico da PUCRS (TECNOPUC). Os entrevistados } \\
\text { serão identificados conforme o papel que desempenham e as atividades que } \\
\text { desenvolvem na Universidade ou no Tecnopuc, sendo então chamados de: (i) } \\
\text { stakeholder universidade 1; (ii) stakeholder Tecnopuc 1, 2 e 3; }\end{array}$ \\
\hline Governo & $\begin{array}{l}\text { O Governo tem a percepção de que para que ocorra desenvolvimento em } \\
\text { nível regional e até mesmo em nível nacional, é necessário subsidiar setores } \\
\text { estratégicos, em ambientes focados em inovação, tais como o TECNOPUC. } \\
\text { Ao investir em parques, o governo articula-se com as Universidades e } \\
\text { setores produtivos alocados no Estado, fomentando o desenvolvimento da } \\
\text { Ciência e Tecnologia. Os entrevistados serão identificados conforme o setor } \\
\text { governamental em que atuam, sendo: (i) governo municipal 1; (ii) governo } \\
\text { estadual 1, 2 e 3. }\end{array}$ \\
\hline \multirow{5}{*}{ Empresas } & $\begin{array}{l}\text { Cobalto - empresa de pequeno porte, atuante somente no mercado nacional. } \\
\text { Seu segmento é voltado, preponderantemente, ao desenvolvimento de } \\
\text { softwares. A empresa possui um produto único, sendo todos os serviços } \\
\text { oferecidos complementares ao seu produto principal. }\end{array}$ \\
\hline & $\begin{array}{l}\text { Lítio - empresa que atua no mercado em nível nacional e internacional, sendo } \\
\text { conhecida em nível global. É vista como uma empresa inovadora que está } \\
\text { em constante transformação. Oferta produtos e serviços de TI com elevada } \\
\text { aplicação de tecnologias de ponta e necessita constantemente atualizar o seu } \\
\text { Centro de P\&D. }\end{array}$ \\
\hline & $\begin{array}{l}\text { Níquel - empresa que atua em nível global, tendo operações em cerca de } \\
170 \text { países no mundo. Caracteriza-se como de grande porte e suas atividades } \\
\text { estão relacionadas diretamente com a inovação em tecnologia. Explora } \\
\text { constantemente como a tecnologia e serviços que oferecem podem ajudar às } \\
\text { empresas a conhecerem seus problemas e desafios. }\end{array}$ \\
\hline & $\begin{array}{l}\text { Silício - empresa que tem atuação global em } 30 \text { países, tendo cerca de } 17.000 \\
\text { colaboradores. Mas sua fundação ocorreu em território nacional, em São Paulo. } \\
\text { Atua com médios e grandes clientes, prestando serviços de TI e provendo de } \\
\text { soluções na área de informática, sendo especializada em tecnologia. }\end{array}$ \\
\hline & $\begin{array}{l}\text { Titânio - empresa que está no mercado há } 16 \text { anos, sem fins lucrativos. É focada } \\
\text { há } 14 \text { anos em um serviço único, sendo este a pesquisa e o desenvolvimento de } \\
\text { serviços de Tl e softwares. }\end{array}$ \\
\hline
\end{tabular}

Fonte: Elaboração própria, a partir dos dados da pesquisa (2016)

O Quadro 2, exposto anteriormente, descreve as principais características e descrições dos três stakeholders analisados nesta pesquisa. A partir dos dados obtidos na coleta, foi desenvolvida a análise dos enfoques dos stakeholders, que está apresentada a seguir.

\section{Análise dos Enfoques dos Stakeholders}

O parque é visto pelo Stakeholder Universidade 1, como um ambiente complexo, com múltiplas possibilidades e interações. Foi caracterizado, segundo um dos entrevistados, como "Um ecossistema de inovação, onde ocorre interação entre diversos atores" (Stakeholder Universidade 1). Na visão da Universidade, estas interações são balizadas, principalmente, pelo segmento acadêmico, em termos de pesquisa e inovação.

Conforme o Stakeholder Tecnopuc 1, em um Parque Tecnológico caracterizado como Científico e Tecnológico, assim como o Tecnopuc, é perceptível um viés muito forte na questão da pesquisa tecnológica. Nesta perspectiva, o parque foi criado pela universidade para fomentar a pesquisa, desenvolvimento e inovação (Stakeholder Tecnopuc 1).

Todo o ambiente e infraestrutura criados e ofertados pelo Parque Tecnológico, como também 
Naiane de Lurdes Santana; Peter Bent Hansen

pela Universidade, constituem um fator que atrai empresas e, consequentemente, uma rede de fornecedores e clientes circulando e interagindo no ambiente do Tecnopuc. Segundo alguns dos entrevistados representantes das empresas instaladas do Tecnopuc (Níquel; Silício e Titânio) o parque oferece um ambiente que compreende: (i) apoio à inovação; (ii) estímulo ao empreendedorismo; (iii) network amplo; (iv) questão logística facilitada; e (v) forte incentivo a P\&D.

Segundo os entrevistados, os parques tecnológicos são uma solução e alternativa de desenvolvimento local e regional, mas o mesmo ainda está muito afastado do ambiente externo da Universidade ou do ambiente onde está instalado. É necessária uma comunicação e interação maior entre o parque e a comunidade que está em sua volta. Ter um contato mais próximo com o meio externo é uma forma proposta pelos entrevistados de atualizar o ecossistema do parque e dos atores envolvidos, para que se desenvolvam atividades e medidas inovadoras de acordo com as reais necessidades da comunidade. De acordo com um dos entrevistados "o problema é que os atores se acomodam em seus papéis e o parque não consegue dar o salto necessário para a realidade atual" (Governo Estadual 1).

Um dos aspectos apontado como relevante, pela bibliografia consultada, e também nas entrevistas, é a oferta de oportunidades de empregos qualificados. Acredita-se que por meio desse aspecto o parque tecnológico se desenvolve e beneficia diretamente a comunidade como um todo, vindo daí uma das suas contribuições social e econômica. Vale salientar, que dentre os indicadores de medição de desempenho propostos nesta pesquisa, este teve concordância de todos os entrevistados, visto como uma forma de atrair pessoas e empresas para estarem envolvidas com o ecossistema do parque tecnológico, podendo estes ter a possibilidade de acesso a cursos de graduação e aperfeiçoamento oferecidos na Universidade, aumentando a renda e a qualidade de vida da comunidade do seu entorno.

Segundo a empresa Cobalto um dos principais benefícios em estar instalada no parque tecnológico são os contratos de Pesquisa e Desenvolvimento (P\&D) estabelecidos em parceria com os Centros de Pesquisa, o que promove para as empresas a exploração e o desenvolvimento das suas capacidades científicas e de pesquisa. O desenvolvimento de capacidades científicas é um fator que pode viabilizar a geração de um produto ou uma nova patente, sendo benéfico para as empresas, principalmente por ter um acesso livre a tal conhecimento no ambiente do Parque, reduzindo os custos na atividade econômica empresarial (empresa Cobalto).

Outro ponto que foi dado destaque pelos entrevistados do Governo foi a dimensão de inovação nos parques tecnológicos. Segundo o Governo Municipal 1:"Inovação é o motor dentro de um parque, fundamentalmente para as empresas de tecnologia de informação. Os entrevistados da Universidade ressaltam que um dos objetivos na relação entre a Universidade e o Parque Tecnológico é a questão do fomento ao empreendedorismo e à inovação. "A universidade tem isso na veia". Uma referência a isso é a criação e operação da Incubadora instalada no parque que promove e acompanha novos empreendedores que possuem um potencial de crescimento já identificado.

Em relação a uma sistemática de mensuração de desempenho de Parques Tecnológicos, os entrevistados destacaram que é essencial a implantação, o acompanhamento e avaliação de resultados dos mesmos. Conforme o Governo Municipal 1: "Quem não mede não gerencia, não acompanha". Porém, o Stakeholder Tecnopuc 1 faz um contraponto quanto a isso "Um sistema de medição de desempenho é importante, mas é muito difícil de montá-lo, se ter em vista que deve ser customizado a cada tipo de parque".

O entrevistado da empresa Lítio expôs a sua visão a respeito de uma proposta de medição de desempenho de parques tecnológicos, afirmando:"Uma sistemática seria outra fonte de informação. Ajudaria bastante no amadurecimento da relação com a universidade". Para a empresa Cobalto "O principal benefício é aumentar a capacidade de tomada de decisão assertiva, reduzindo os riscos das empresas, reduzindo o desalinhamento entre as ações das mesmas com os próprios objetivos".

Todos os entrevistados identificaram, em pelo menos algum aspecto específico, a necessidade e importância de mensurar o desempenho de parques tecnológicos, de ter uma sistemática de avaliação de desempenho que possibilite o efetivo acompanhamento do desenvolvimento desses parques. Apresenta-se a seguir, nos Quadros 3 e 4, uma síntese dos resultados obtidos a partir das entrevistas realizadas neste estudo, tendo a perspectiva dos stakeholders a respeito da definição (entendimento do que é um parque tecnológico) e dos objetivos no vínculo com o parque tecnológico. 
Quadro 3. Síntese das definições de Parques Tecnológicos segundo os Stakeholders entrevistados

\begin{tabular}{|l|l|}
\hline Stakeholder & \multicolumn{1}{c|}{ Definição de Parques Tecnológicos } \\
\hline Universidade & $\begin{array}{l}\text { O parque tecnológico é um ecossistema de inovação, que é permeado pelo } \\
\text { conceito de tríplice hélice. O parque é o responsável por promover e gerir as } \\
\text { interações entre os atores da relação, tendo em vista a sinergia dos mesmos e o } \\
\text { desenvolvimento científico-tecnológico da região. É um ambiente de múltiplas } \\
\text { possibilidades e interações. }\end{array}$ \\
\hline Governo & $\begin{array}{l}\text { Parques tecnológicos são estruturas baseadas na tríplice hélice. O parque é um } \\
\text { ambiente gerador e promotor de inovação, onde as empresas podem colocar } \\
\text { suas demandas e potencializá-las por meio de pesquisa básica e aplicada. }\end{array}$ \\
\hline Empresa & $\begin{array}{l}\text { O parque tecnológico é um ambiente que visa à integração e cooperação } \\
\text { entre as empresas e a universidade. É um catalisador de diversos tipos de } \\
\text { conhecimento. Trata-se de uma iniciativa que visa à criação de ambientes de } \\
\text { inovação que promova desenvolvimento de novas soluções e produtos. }\end{array}$ \\
\hline
\end{tabular}

Fonte: Elaboração própria, a partir dos dados da pesquisa (2016)

No Quadro 4 estão listados os objetivos dos stakeholders ao vincular-se ao Tecnopuc. Esses são os objetivos apontados como essenciais e relevantes de acordo com a percepção dos entrevistados nesta pesquisa. Vale mencionar que desde o seu formato inicial houve alterações na redação/ explicitação desses objetivos estratégicos seguindo as sugestões dos stakeholders entrevistados.

Quadro 4. Objetivos dos stakeholders ao vincular-se ao parque tecnológico

\begin{tabular}{|l|l|}
\hline Stakeholder & \multicolumn{1}{c|}{ Objetivos Estratégicos } \\
\hline \multirow{5}{*}{ Universidade } & $\begin{array}{l}\text { 1- Encorajar o surgimento de empresas spin-off iniciadas por acadêmicos, } \\
\text { prestando serviços para a Universidade e outras empresas localizadas no Parque } \\
\text { Tecnológico. }\end{array}$ \\
\cline { 2 - 3 } & $\begin{array}{l}\text { 2- Facilitar a transferência de tecnologia entre instituições acadêmicas e } \\
\text { empresas localizadas no parque tecnológico. }\end{array}$ \\
\cline { 2 - 3 } & $\begin{array}{l}\text { 3- Aumentar a relevância das pesquisas acadêmicas para as empresas e a } \\
\text { sociedade. }\end{array}$ \\
\hline \multirow{5}{*}{ Governo } & $\begin{array}{l}\text { 4- Beneficiar-se de alianças estratégicas em nível regional, nacional e } \\
\text { internacional, estimulando a formação de novas empresas de base tecnológica. }\end{array}$ \\
\cline { 2 - 3 } & $\begin{array}{l}\text { 5- Atrair e destinar investimentos para atividades de P\&D, promovendo } \\
\text { equilíbrio regional em capacidade, inovação e investimento. }\end{array}$ \\
\cline { 2 - 3 } & $\begin{array}{l}\text { 6- Gerar novos postos de trabalho na região, melhorando a performance da } \\
\text { economia local. }\end{array}$ \\
\hline \multirow{5}{*}{ Empresas } & $\begin{array}{l}\text { 7- Gerar oportunidades de consultoria e aprendizagem, recrutando } \\
\text { pesquisadores e estudantes acadêmicos. }\end{array}$ \\
\cline { 2 - 3 } & $\begin{array}{l}\text { 8- Estabelecer contratos de P\&D com os Centros de Pesquisa disponibilizados } \\
\text { no Parque Tecnológico, desenvolvendo e explorando capacidades científicas. }\end{array}$ \\
\cline { 2 - 3 } & $\begin{array}{l}\text { 9- Por meio dos recursos disponibilizados no Parque Tecnológico, desenvolver e } \\
\text { aperfeiçoar novos produtos e serviços inovadores. }\end{array}$ \\
\cline { 2 - 3 } & $\begin{array}{l}\text { 10- Estabelecer sinergia e cooperação entre as empresas do parque tecnológico, } \\
\text { resultando em benefícios mútuos. }\end{array}$ \\
\hline
\end{tabular}

Fonte: Elaboração própria, a partir dos dados da pesquisa (2016)

Tendo como ponto de partida os resultados apresentados anteriormente, foram desenvolvidos os principais elementos de um sistema de indicadores para a avaliação de desempenho de parques tecnológicos, a partir da perspectiva do TECNOPUC. A proposta está apresentada na próxima seção. 


\section{PROPOSTA DE UM SISTEMA DE AVALIAÇÃO DE DESEMPENHO PARA PARQUES TECNOLÓGICOS}

Através da identificação dos objetivos dos stakeholders ao vincularem-se ao parque tecnológico e juntamente com a fundamentação teórica adotada neste trabalho, foram propostos indicadores que permitissem mensurar o desempenho de parques tecnológicos. Por meio das entrevistas realizadas foi possível identificar, sob o ponto de vista dos stakeholders, quais indicadores são viavelmente mensuráveis e relevantes para a avaliação de desempenho em parques tecnológicos.

A partir das afinidades entre o que disseram os stakeholders entrevistados foram agrupados os objetivos que possuíam um grau próximo de interação e seus respectivos indicadores, estabelecendose "Dimensões", existentes no ecossistema do Parque Tecnológico, as quais foram identificadas principalmente através dos dados obtidos na análise de conteúdo das entrevistas.

Ao realizar-se a atividade de agrupamento, através do Diagrama de Afinidades (Moura, 1994), pôde-se perceber a constante interação e comunicação entre as ações e decisões dos stakeholders. Portanto, o desenvolvimento e funcionamento do Parque Tecnológico dependem da integração e estratégias conjuntas. No sistema de avaliação de desempenho de parques tecnológicos proposto nesta pesquisa, foram identificadas e estruturadas quatro dimensões principais, que foram evidenciadas durante a aplicação da técnica do Diagrama de Afinidades, sendo estas: (i) Dimensão Tecnológica; (ii) Dimensão Social; (iii) Dimensão Acadêmica; e (iv) Dimensão Econômica. Na dimensão tecnológica a interação de objetivos destacou-se entre os stakeholders Empresas e a Universidade. Nesta interação a universidade é o meio que promove a transferência de conhecimentos, assim como propicia os recursos disponibilizados no parque tecnológico e no campus acadêmico. As empresas constituem a parte que anseia por ter acesso a tais recursos, de forma que estes sejam ferramentas de aprimoramento para os seus serviços e/ou produtos.

A dimensão social está vinculada à interação do Governo e Empresas. O governo tem o papel de promover desenvolvimento e crescimento, e necessita de empresas instaladas e com forte atuação na região. Empresas que sejam uma fonte de recursos, tanto por meio de impostos, assim como também através do recrutamento e aperfeiçoamento da mão de obra. As empresas por sua vez, também buscam estabelecer sinergia e cooperação com o setor governamental, visando benefícios e apoios para que obtenham condições para expandir ou estruturar centros de produção e desenvolvimento tecnológico.

Na dimensão acadêmica a interação está fortemente vinculada na comunicação e integração entre as Empresas e a Universidade. Verificou-se que as empresas, desde o seu contato inicial com o Parque Tecnológico, vinculam-se e estabelecem parcerias com a academia. Parcerias estas, orientadas para o desenvolvimento e exploração das capacidades científicas dentro do ambiente empresarial. A integração entre a Academia e as Empresas impacta diretamente na relevância das pesquisas realizadas, pois as relações entre teoria e prática desenvolvidas em contextos reais permitem aferições mais concretas e a possibilidade de identificar e propor soluções, tanto para problemas teóricos como práticos. Para o empresário é destacado como benéfico que a pesquisa e/ou consultoria do pesquisador dentro do seu ambiente possibilitem uma visão diferenciada e especializada, esta capaz de impactar o crescimento e a revitalização empresarial.

A dimensão econômica, na estrutura proposta neste trabalho, é a única dimensão onde foi identificada a interação e percepção de todos os stakeholders, sendo vínculo entre a Universidade, o Governo e as Empresas. A questão econômica apresenta fatores que impactam todos os atores. A universidade está vinculada à dimensão econômica devido ao encorajamento de empresas por meio da Incubadora, as quais podem eventualmente prestar posteriormente serviço também para a Academia e as demais empresas existentes no ambiente do parque. O governo, diante deste contexto, estimula as alianças que são meios estratégicos na criação e manutenção de empresas iniciantes, principalmente as de base tecnológica. Como resultado econômico, o desenvolvimento e crescimento de empresas é um motor para o desenvolvimento da região, principalmente em torno do parque tecnológico, devido ao ecossistema que foi desenvolvido, permitindo a melhoria de imagem da base industrial local - conforme destacado pelos stakeholders do Governo, principal peça interessada nos fatores anteriormente citados. Os atores desempenham atividades conjuntas que captam e promovem a atração de investimentos nos setores econômicos do parque, de tal forma que todos usufruam dos recursos e meios disponibilizados, resultando em benefícios mútuos.

A Figura 1 retrata as dimensões até então descritas, assim como a interação entre os stakeholders do parque tecnológico. 


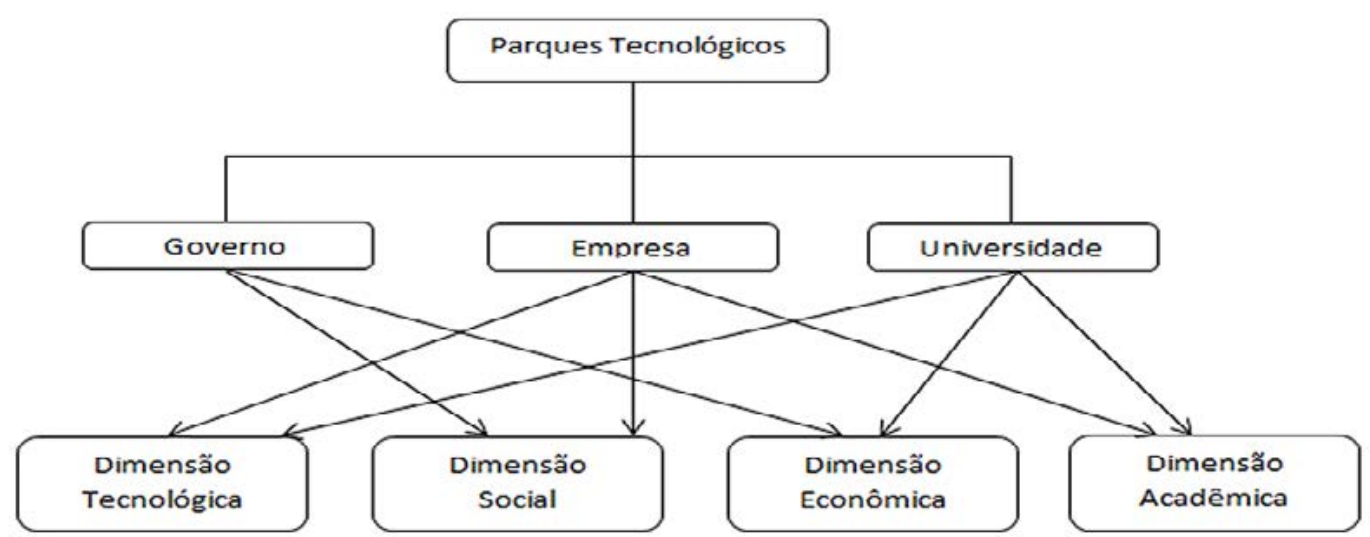

Figura 1- Interação entre os Stakeholders do Parque Tecnológico

Fonte: Elaboração própria, a partir dos dados da pesquisa (2016)

Os indicadores que foram selecionados para a proposta apresentada (Quadro 5), seguiram critérios como a concordância de pelo menos a metade do grupo dos entrevistados de cada stakeholder específico e, assim, foram vinculados aos objetivos estratégicos.

Quadro 5. Proposta do Sistema de Avaliação de Desempenho em Parques Tecnológicos

\begin{tabular}{|c|c|c|}
\hline Dimensão & Objetivos Estratégicos & Indicadores \\
\hline \multirow{5}{*}{ 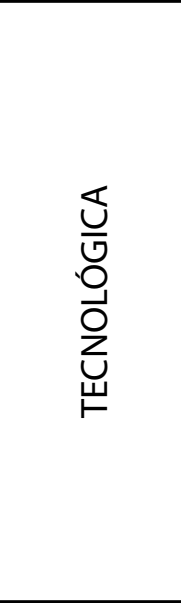 } & \multirow{3}{*}{$\begin{array}{l}\text { 1- Facilitar a transferência de } \\
\text { tecnologia entre instituições } \\
\text { acadêmicas e empresas } \\
\text { localizadas no parque } \\
\text { tecnológico. }\end{array}$} & $\begin{array}{l}1.2 \mathrm{n}^{\circ} \text { de resultados obtidos através da interação } \\
\text { dos stakeholders do parque com os centros de } \\
\text { tecnologia da comunidade científica. }\end{array}$ \\
\hline & & $\begin{array}{l}1.3 n^{\circ} \text { de transferências de tecnologia e } \\
\text { conhecimento entre academia e empresa. }\end{array}$ \\
\hline & & $\begin{array}{l}1.4 \mathrm{n}^{\circ} \text { de avanços e desenvolvimentos tecnológicos } \\
\text { que foram aplicados internamente nas empresas e } \\
\text { academia. }\end{array}$ \\
\hline & \multirow{2}{*}{$\begin{array}{l}\text { 2- Por meio dos recursos } \\
\text { disponibilizados no Parque } \\
\text { Tecnológico, desenvolver e } \\
\text { aperfeiçoar novos produtos e } \\
\text { serviços inovadores. }\end{array}$} & $\begin{array}{l}2.1 \mathrm{n}^{\circ} \text { de resultados de pesquisas comercializados e } \\
\text { divulgados. }\end{array}$ \\
\hline & & $\begin{array}{l}2.2 n^{\circ} \text { de participações em eventos científico- } \\
\text { tecnológicos. }\end{array}$ \\
\hline \multirow{6}{*}{ 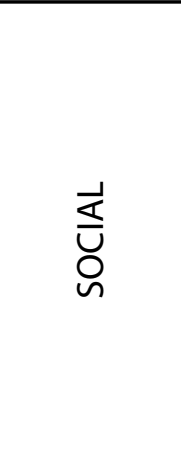 } & \multirow{3}{*}{$\begin{array}{l}\text { 3- Gerar novos postos } \\
\text { de trabalho na região, } \\
\text { melhorando a performance da } \\
\text { economia local. }\end{array}$} & 3.1 grau de impacto na renda da comunidade local. \\
\hline & & $\begin{array}{l}3.2 \text { quantidade de mão de obra qualificada e } \\
\text { treinada. }\end{array}$ \\
\hline & & $\begin{array}{l}3.3 \mathrm{n}^{\circ} \text { de postos de trabalho formal e informais } \\
\text { gerados. }\end{array}$ \\
\hline & \multirow{3}{*}{$\begin{array}{l}\text { 4- Estabelecer sinergia e } \\
\text { cooperação entre as empresas } \\
\text { do parque tecnológico, } \\
\text { resultando em benefícios } \\
\text { mútuos. }\end{array}$} & $\begin{array}{l}4.1 n^{\circ} \text { de redes criadas e estabelecidas com outras } \\
\text { empresas do parque. }\end{array}$ \\
\hline & & $4.2 n^{\circ}$ de projetos conjuntos financiados. \\
\hline & & 4.3 acordos conjuntos de pesquisa divulgados. \\
\hline
\end{tabular}




\begin{tabular}{|c|c|c|}
\hline \multirow{11}{*}{ 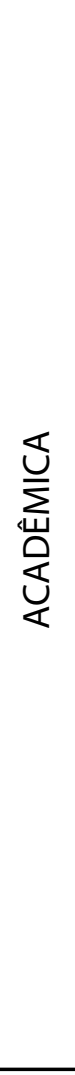 } & \multirow{2}{*}{$\begin{array}{l}\text { 5- Aumentar a relevância das } \\
\text { pesquisas acadêmicas para as } \\
\text { empresas e a sociedade. }\end{array}$} & $\begin{array}{l}5.1 \mathrm{n}^{\circ} \text { de resultados de pesquisas científicas } \\
\text { comercializados e divulgados. }\end{array}$ \\
\hline & & $\begin{array}{l}5.2 n^{\circ} \text { de artigos/papers divulgados em eventos } \\
\text { científico-tecnológicos. }\end{array}$ \\
\hline & \multirow{5}{*}{$\begin{array}{l}\text { 6- Estabelecer contratos } \\
\text { de P\&D com os Centros de } \\
\text { Pesquisa disponibilizados } \\
\text { no Parque Tecnológico, } \\
\text { desenvolvendo e explorando } \\
\text { capacidades científicas. }\end{array}$} & $\begin{array}{l}6.1 \mathrm{n}^{\circ} \text { de competências e capacidades científicas } \\
\text { desenvolvidas. }\end{array}$ \\
\hline & & $\begin{array}{l}6.2 \mathrm{n}^{\circ} \text { de projetos e pesquisas científicas } \\
\text { produzidos. }\end{array}$ \\
\hline & & $\begin{array}{l}6.3 \mathrm{n}^{\circ} \text { de contratos estabelecidos com a instituição } \\
\text { acadêmica. }\end{array}$ \\
\hline & & $\begin{array}{l}6.4 n^{\circ} \text { de bolsas destinadas a formação de } \\
\text { pesquisadores. }\end{array}$ \\
\hline & & $\begin{array}{l}6.5 \text { quantidade de pesquisa transformada em novos } \\
\text { produtos. }\end{array}$ \\
\hline & \multirow{4}{*}{$\begin{array}{l}\text { 7- Gerar oportunidades de } \\
\text { consultoria e aprendizagem, } \\
\text { recrutando pesquisadores e } \\
\text { estudantes acadêmicos. }\end{array}$} & $\begin{array}{l}7.1 \text { quantidade de reuniões e consultorias } \\
\text { realizadas. }\end{array}$ \\
\hline & & $\begin{array}{l}7.2 \text { quantidade de novas competências definidas e } \\
\text { identificadas. }\end{array}$ \\
\hline & & $\begin{array}{l}7.3 \text { quantidade de novos conhecimentos } \\
\text { adquiridos, desenvolvidos e compartilhados. }\end{array}$ \\
\hline & & $\begin{array}{l}7.4 \mathrm{n}^{\circ} \text { de bolsas ofertadas a estudantes } \\
\text { universitários. }\end{array}$ \\
\hline \multirow{7}{*}{ 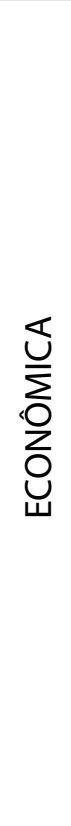 } & $\begin{array}{l}\text { 8- Encorajar o surgimento de } \\
\text { empresas spin-off iniciadas } \\
\text { por acadêmicos, prestando } \\
\text { serviços para a Universidade } \\
\text { e outras empresas localizadas } \\
\text { no Parque Tecnológico. }\end{array}$ & $\begin{array}{l}8.1 \mathrm{n}^{\circ} \text { de empresas incubadas recém-ingressas no } \\
\text { mercado. }\end{array}$ \\
\hline & \multirow{2}{*}{$\begin{array}{l}\text { 9- Beneficiar-se de alianças } \\
\text { estratégicas em nível regional, } \\
\text { nacional e internacional, } \\
\text { estimulando a formação de } \\
\text { novas empresas de base } \\
\text { tecnológica. }\end{array}$} & $\begin{array}{l}9.1 \mathrm{n}^{\circ} \text { de empresas de base tecnológica criadas e } \\
\text { incubadas. }\end{array}$ \\
\hline & & $\begin{array}{l}9.2 n^{\circ} \text { de projetos e pesquisas realizados através de } \\
\text { alianças estratégicas. }\end{array}$ \\
\hline & \multirow{4}{*}{$\begin{array}{l}\text { 10- Atrair e destinar } \\
\text { investimentos para atividades } \\
\text { de } P \& D \text {, promovendo } \\
\text { equilíbrio regional em } \\
\text { capacidade, inovação e } \\
\text { investimento. }\end{array}$} & $\begin{array}{l}10.1 \text { volume de recursos obtidos para fomentar } \\
\text { pesquisas universitárias e empresariais. }\end{array}$ \\
\hline & & $10.2 n^{\circ}$ bolsas de pesquisa ofertadas. \\
\hline & & $10.3 n^{\circ}$ de contratos de financiamento formalizados. \\
\hline & & $\begin{array}{l}\text { 10.4 Porcentagem de retorno sobre o capital } \\
\text { investido. }\end{array}$ \\
\hline
\end{tabular}

Fonte: Elaboração própria, a partir dos dados da pesquisa (2016)

Conforme apresentado no Quadro 5, o sistema de avaliação de desempenho proposto neste trabalho, a partir do estudo realizado com stakeholders do Tecnopuc, ficou composto de 4 dimensões (tecnológica, social, acadêmica e econômica) nas quais estão vinculados 10 objetivos estratégicos e 29 indicadores considerados relevantes para avaliação de desempenho de parques científicos e tecnológicos. 


\section{CONCLUSÕES}

O intuito inicial desta pesquisa foi construir uma sistemática de avaliação de desempenho que englobasse os diferentes objetivos estratégicos dos parques tecnológicos sob a percepção dos seus principais stakeholders, dentro de um ecossistema delineado por constantes interações e com um alto grau de empreendedorismo e inovação.

Este trabalho, portanto, atingiu o seu principal objetivo ao desenvolver um conjunto de indicadores - a partir das diversas expectativas e visões diferentes sobre o parque focalizado - os quais podem ser úteis para avaliar o desempenho de parques tecnológicos, considerando várias dimensões que inter-relacionam objetivos dos stakeholders da tríplice hélice (Universidade- GovernoIndústria).

A partir deste estudo, identificou-se que existem objetivos bem particulares dos stakeholders, assim como há um aparato amplo de expectativas em comum ou que se inter-relacionam. Diante deste contexto, entende-se que os atores do parque estudado (o Tecnopuc) estabelecem uma relação de sinergia e cooperação e que esperam benefícios advindos dessa relação. A interação entre os stakeholders viabilizou o estabelecimento dos indicadores, que foram destacados como pontoschave para a avaliação do desempenho do parque.

Devido ao fato do instrumento de pesquisa ter sido desenvolvido no ambiente do Tecnopuc, caracterizado como "parque científico e tecnológico", a proposta de avaliação de desempenho é desenhada segundo as esferas atuantes de tal estrutura, tendo-se principalmente uma visão acadêmica. Neste sentido, sugere-se que futuros estudos investiguem a aplicação e/ou adequação dessa proposta (conjunto de indicadores e suas correspondentes dimensões) em outros tipos de parques, localizados em diversas partes do Brasil. Segundo os stakeholders entrevistados, a mensuração de desempenho do parque frente aos diversos interesses existentes, permite uma forma de avaliação do seu grau de atuação e do seu potencial de crescimento. Potencial este que, de forma significativa, foi destacado pelos entrevistados como um campo muito além "dos muros" da Instituição Acadêmica.

Os resultados deste trabalho apontam que a gestão de parques tecnológicos requer, além de indicadores confiáveis, também, uma atuação próxima e contínua com os stakeholders envolvidos e atuantes neste contexto, refletindo em benefícios e impactos na sociedade como um todo. 


\section{REFERÊNCIAS}

Abdalla, M. M., Calvosa, M. V. D., \& Batista, L. G. (2009). Hélice Tríplice no Brasil: um ensaio teórico acerca dos benefícios da entrada da universidade nas parcerias estatais. Revista Cadernos de Administração da Faculdade Salesiana Maria Auxiliadora, 1(3).

ABDI - Associação Brasileira de Desenvolvimento Industrial. (2008). Parques tecnológicos no Brasil: Estudo, análise e proposições. Brasília: ABDI/ANPROTEC.

Atrasas, A. L., de Souza Dias, J. M. C., de Sousa Leite, L. A., \& Brasília, D. F. (2003). Parques tecnológicos e incubadoras de empresas: fatores de desenvolvimento tecnológico e regional em Portugal e Espanha. Relatório de Viagem Internacional.

Bardin, L. (2006). Análise de conteúdo. Lisboa: Edições 70.

Campanella, F., Della Peruta, M.R, \& Del Giudice, M. (2014). Creating conditions for innovative performance of science parks in Europe. Journal of Intellectual Capital, 15(4), 576-596.

Carpinetti, L. C., Galdámez, E. V. C., \& Gerolamo, M. C. (2007). A measurement system for managing performance of industrial clusters: A conceptual model and research cases. Paper presented at 14th International EurOMA Conference, Ankara, Turkey.

Chan, K. Y. A., Oerlemans, L. A., \& Pretorius, M. W. (2011). Innovation outcomes of South African new technology-based firms: A contribution to the debate on the performance of science park firms. South African Journal of Economic and Management Sciences, 14(4), 361-378.

Chikán, A. (2008) National and firm competitiveness: a general research model. Competitiveness Review: An International Business Journal Incorporating Journal of Global Competitiveness, 18(1/2).

Chiochetta, J. C. (2010). Proposta de um modelo de governança para parques tecnológicos (Tese de Doutorado, Universidade Federal do Rio Grande do Sul). Recuperado de: http:// www.producao.ufrgs.br/arquivos/publicacoes/348_TESE\%20-\%20CHIOCHETTA-JC\%20-\%20 HOMOLOGA\%C3\%87\%C3\%830\%20-\%20revista\%2014-01.pdf

Eler, E. O. \& Andalecio, A. M. L. (2015). Indicadores de inovação: Estudo comparativo entre o Brasil e os demais países dos BRICS. GEINTEC: Gestão, Inovação e Tecnologias, 5(1), 1683-1702.

Esposto, K. F., Gerolamo, M. C., \& Rentes, A. F (2002). Sistema de medição de desempenho: Uma proposta conceitual. Artigo apresentado no $22^{\circ}$ Encontro Nacional de Engenharia de Produção. Curitiba, Brasil.

Etzkowitz, H. (2009). Hélice Tríplice: Universidade, indústria e governo: Inovação em movimento. Porto Alegre: EDIPUCRS.

Etzkowitz, H., \& Leydesdorff, L. (1995). The Triple Helix of university-industry-government relations: A laboratory for knowledge based economic development. EASST Review, 14(1), 14-19.

Etzkowitz, H., \& Leydesdorff, L. (1997). Universities and the Global Economy: A triple helix of universityindustry-government relations. London: Cassell Academy.

Etzkowitz, H., \& Leydesdorff, L. (2000). The dynamics of innovation: From National System and "Mode 2" to a Triple Helix of University industry government relation. Research Policy, 29(2), 109123.

Fernandes, S. C. R. (2014) Avaliação de parques tecnológicos: Uma proposta de modelo para parques de $3^{\circ}$ geração (Dissertação de Mestrado, Universidade Federal do Rio de Janeiro). Recuperado de http://www.coppead.ufrj.br/upload/publicacoes/Sheila_Fernandes.pdf 
Galdámez, E. V. C.; Carpinetti, L. C. R.; \& Gerolamo, M. C. (2009). Proposta de um sistema de avaliação do desempenho para arranjos produtivos locais. Revista Gestão \& Produção, 16(1).

Giugliani, E., Selig, P. M., \& dos Santos, N. (2012). Modelo de governança para parques científicos e tecnológicos: Análise e contexto. Trabalho apresentado no $22^{\circ}$ Seminário Nacional de Parques Tecnológicos e Incubadoras de Empresas e XX Workshop Anprotec, Foz do Iguaçu, Paraná.

Guerra, A. F., Figueiredo, M. L., \& Ruscheinsky, A. (2015). Ambiente e sustentabilidade no sistema nacional de ciência, tecnologia e inovação do Brasil. Revista Iberoamericana Universitaria en Ambiente, Sociedad y Sustentabilidad, 1(1), 125-138.

IASP - International Association of Science Parks. Science Park. IASP International Board, Fev., 2002. Disponível em: http://www.iasp.ws/publico/index.jsp?enl=1.

Jung Neto, R. J. \& de Paula, E. A. W. (2009). Indicadores de avaliação de desempenho para o Parque Científico e Tecnológico da PUCRS-TECNOPUC, na percepção de seus principais "stakeholders". Trabalho apresentado no $3^{\circ}$ Fórum Global de Inovação e Empreendedorismo, Florianópolis, Santa Catarina.

Kiyan, F. M. (2001). Proposta para o desenvolvimento de indicadores de desempenho como suporte estratégico (Dissertação de Mestrado, Universidade de São Paulo). Recuperado de http://www. teses.usp.br/teses/disponiveis/18/18140/tde-02082002-075900/pt-br.php

Lima, R. H. P., Marinho, C. A., \& Carpinetti, L. C. R. (2011). Minimizando as barreiras para a medição de desempenho em arranjos produtivos locais: $\mathrm{O}$ caso do Sertãozinho. Revista Brasileira de Estratégia, 4(2).

Massey, D., Quintas, P., \& Wield, D. (1992). High-Tech Fantasies: Science parks in society. London: Routledge.

Mazzarolo, C. F. (2010). Estratégia de apoio à inovação em tecnologias da informação no parque tecnológico Capital Digital (Dissertação de Mestrado, Universidade de Brasília). Recuperado de http://repositorio.unb.br/handle/10482/8259

MDIC (2004). O Futuro da Indústria de software: A perspectiva do Brasil. Brasília: Ministério do Desenvolvimento, Indústria e Comércio Exterior.

Medori, D. \& Steeple, D. (2000) A framework for auditing and enhancing performance measurement systems. International Journal of Operations \& Production Management, 20(5), 520-533.

Moura, E. (1994). As sete ferramentas gerenciais da qualidade: Implementação e melhoria contínua com maior eficácia. São Paulo, MAKRON Books.

Neves, J. L. (1996). Pesquisa qualitativa: características, usos e possibilidades. Caderno de Pesquisa em Administração, 1(3).

Parung, J., \& Bititci, U. S. (2006). A conceptual metric for managing collaborative networks. Journal of Modelling in Management, 2(1), 116-136.

Ribeiro, M. L., Botelho, S.S.C., \& Duarte Filho, N. (2012). Avaliando a estratégia do Parque Tecnológico Oceantec a partir da utilização da ferramenta Estrategigrama. Trabalho apresentado no Simpósio de Excelência em Gestão e Tecnologia, Rio de Janeiro.

Spinola, M. M., \& Pessôa, M. S. P. (1997). Tecnologia da Informação. In Contador, J. C. (Coord.), Gestão de operações. São Paulo: Edgar Blücher.

Spolidoro, R., \& Audy, J. (2008). Parque Científico e Tecnológico da PUCRS. Porto Alegre: Edipucrs. 
Steiner, J. E., Cassim, M. B., \& Robazzi, A.C. (2008). Parques tecnológicos: Ambientes de inovação. São Paulo: Instituto de Estudos Avançados da Universidade de São Paulo.

Tezza, R., Bornia, A. C., \& Vey, I. H. (2010). Sistemas de medição de desempenho: Uma revisão e classificação da literatura. Gestão \& Produção, 17(1), 75-93.

Vedovello, C. (2000). Aspectos relevantes de parques tecnológicos e incubadores de empresas. Revista do BNDES, 7(14).

Yin, R. K. (2015). Estudo de caso: Planejamento e métodos. Porto Alegre: Bookman.

Zouain, D. M. \& Plonski, G. A. (2006) Parques tecnológicos: planejamento e gestão. Brasília: Anprotec/ Sebrae. 